\begin{tabular}{|c|l|}
\hline Title & Attention-spreading Based on Hierarchical Spatial Representations for Connected Objects \\
\hline Author(s) & Kasai, Tetsuko \\
\hline Citation & Journal of Cognitive Neuroscience, 22(1), 12-22 \\
\hline https:/doi.org/L0.1162/0cn.2008.21158
\end{tabular}

Instructions for use 


\title{
Attention-spreading Based on Hierarchical Spatial Representations for Connected Objects
}

\author{
Tetsuko Kasai
}

\begin{abstract}
Attention selects objects or groups as the most fundamental unit, and this may be achieved through a process in which attention automatically spreads throughout their entire region. Previously, we found that a lateralized potential relative to an attended hemifield at occipito-temporal electrode sites reflects attentionspreading in response to connected bilateral stimuli [Kasai, T., \& Kondo, M. Electrophysiological correlates of attention-spreading in visual grouping. NeuroReport, 18, 93-98, 2007]. The present study examined the nature of object representations by manipu-
\end{abstract}

\section{INTRODUCTION}

Consistent with the fact that we interact with objects in everyday life, extensive research has shown that the most fundamental unit of attentional selection is objects or groups, which are determined by Gestalt principles such as adjacency or similarity in the visual field (for reviews, see Hopf, Schoenfeld, \& Heinze, 2005; Scholl, 2001; Driver \& Baylis, 1998). Although various tasks have been used to show this effect (e.g., spatial cueing, flanker task, divided-attention task), participants are generally required to pay attention to a particular spatial location or feature within an object, and behavioral or neural responses to other spatial regions or features that belong to that object are also facilitated, although the object is completely task-irrelevant. Such object-based spatial or feature selection may be achieved through obligatory attention-spreading over the entire representation for the object. By revealing the properties or mechanisms of such attention-spreading, we may contribute to understanding how we realize rapid and effortless cognition and action toward objects in a fragmented visual environment.

For this objective, event-related potentials (ERPs) can offer particularly useful information (e.g., Busse, Roberts, Crist, Weissman, \& Woldorff, 2005; Schoenfeld et al., 2003; Weber, Kramer, \& Miller, 1997). For example, by using experimental paradigms consistent with the typical spatial cueing task at the corner of previewed objects in behavioral studies (Egly, Driver, \& Rafal, 1994), some

Hokkaido University, Sapporo, Japan lating the extent of grouping through connectedness, while controlling the symmetrical structure of bilateral stimuli. The electrophysiological results of two experiments consistently indicated that attention was guided twice in association with perceptual grouping in the early phase (N1, 150-200 msec poststimulus) and with the unity of an object in the later phase (N2pc, 310/330 $390 \mathrm{msec}$ ). This suggests that there are two processes in objectbased spatial selection, and these are discussed with regard to their cognitive mechanisms and object representations.

studies have shown that the early N1 component (at 140$180 \mathrm{msec}$ poststimulus), in response to the onset or offset of a target or target-related feature, enlarged when it appeared at an unattended location on the object to which the attended location belonged compared to when it appeared at an unattended location on another object (Martinez, Ramanathan, Foxe, Javitt, \& Hillyard, 2007; Martinez, Teder-Salejarui, \& Hillyard, 2007; Martinez et al., 2006; He, Fan, Zhou, \& Chen, 2004). Because spatial attention modulates P1 and N1 amplitudes, this finding indicates that spatial and object-based attention are, at least partly, based on a common early cortical mechanism. This supports the notion that has been addressed in behavioral studies, that is, "object" is fundamentally a spatial representation such as a "grouped array of locations" (e.g., Vecera, 1994). Whereas an fMRI study has also supported the object-based spatial selection view (Muller \& Kleinschmdt, 2003), ERP studies offer precise timing information. Interestingly, the early object-based N1 effect was perceptual, rather than physical, because it was seen for illusory objects (Martinez, Ramanathan, et al., 2007; Martinez, Teder-Salejarui, et al., 2007).

The present study describes the timing of object-based spatial selection in response to the onset of an object or a group itself and its nature for object representation. Using a sustained-focal-attention task that involved bilateral stimulus arrays (Woldorff et al., 2002; Heinze et al., 1994; Heinze, Luck, Mangun, \& Hillyard, 1990), Kasai and Kondo (2007) explored electrophysiological correlates of attention-spreading over objects that were grouped by connecting lines. Although the participants attended to one hemifield, connected or unconnected bilateral 
stimuli were rapidly presented in random order. The task was to press a button if the infrequent target (i.e., rectangle) appeared at an attended hemifield among a sequence of standard stimuli (square). The direction of spatial attention was indexed by a larger amplitude of posterior ERPs at the hemisphere contralateral, rather than ipsilateral, to the attended hemifield. Transient attentionspreading was observed only for connected target stimuli, which was reflected by an N2pc (posterior-contralateral) component at 190-250 msec after stimulus onset. Because N2pc has been used as a marker of the momentby-moment direction of spatial attention (e.g., Woodman \& Luck, 1999), it can be a useful index for the timing of attention-spreading for various defining factors of objects/ group.

$\mathrm{N} 2 \mathrm{pc}$ is associated with the suppression of competing stimuli (e.g., Hopf, Boelmans, Schoenfeld, Heinze, \& Luck, 2002; Luck \& Hillyard, 1994), so that the finding that attention-spreading was associated with N2pc is consistent with the biased competition model, in which visual grouping or object structure is a bottom-up factor to bias competitive interactions in visual cortex (e.g., Desimone \& Duncan, 1995). Here, object representations may be constructed step by step from retinal input (e.g., Marr, 1982) via highly parallel-hierarchical structures of visual cortex. However, the level or representation of objectprocessing that is the basis for attention-spreading remains unclear. For example, extensive studies have undiscriminated object from group (e.g., Driver \& Baylis, 1998): Connectedness is the most fundamental factor not only for creating a single object or homogenous region but also for perceptual grouping (Palmer, 2003; Watson \& Kramer, 1999). However, perceptual grouping does not require that the group is a single object, that is, two discrete objects or regions can be considered grouped when they have common features, such as color, shape, or motion.

The main purpose of the present study was to examine whether the manifestation of attention-spreading in ERPs is associated with perceptual grouping or with object unity in terms of connectedness. In two experiments which used an experimental paradigm similar to that described by Kasai and Kondo (2007), three stimulus conditions were set: unconnected condition, weakly connected condition, and strongly connected condition (see Figure 1). The important point is that the unconnected condition included two objects, whereas the two connected conditions included only one object. The extent of perceptual grouping can be assumed to gradually increase among the three conditions. If attention spreads based on grouping, or if there is no need to distinguish between group and object, the attention effects of ERPs should vary as the extent of grouping increases. In contrast, if attention spreads based on object unity, attention effects should decrease for the connected conditions equally. In addition, a minor purpose of the present study was to test whether attentionspreading was specific to the target with behavioral performance, as the symmetrical structure (i.e., a grouping factor) of the standard bilateral stimuli might have caused a ceiling effect in the previous study (Kasai \& Kondo, 2007).

\section{EXPERIMENT 1}

This experiment examined lateralized attention effects in ERPs in response to connected objects, where the extent of connectedness was varied by the thickness of the connecting line (Figure 1). The task was to respond when the target square was presented at an attended

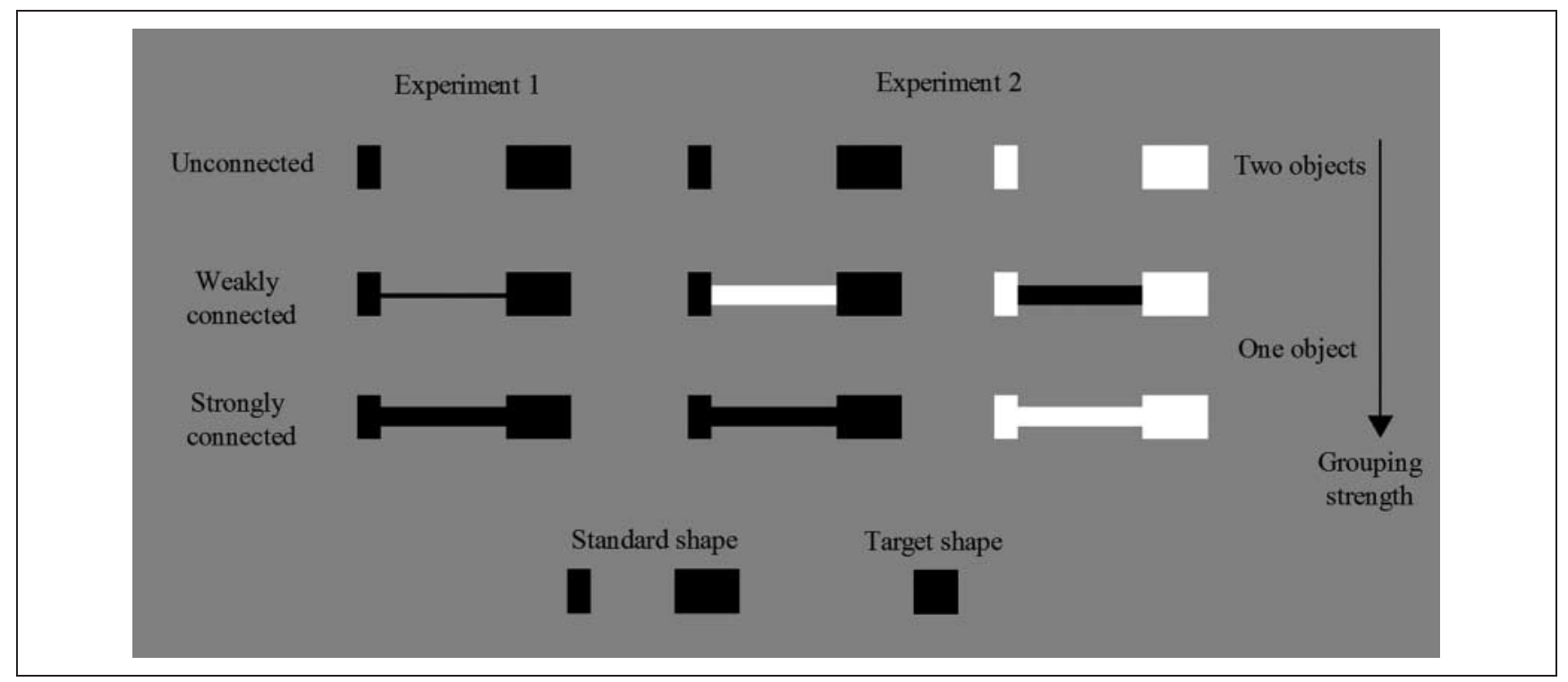

Figure 1. Bilateral stimuli used in Experiments 1 and 2. 
hemifield, and the standard bilateral stimuli had a more asymmetric structure.

\section{Methods}

\section{Participants}

Ten volunteers ( 5 women), aged 21 to 33 years (mean = 25.8 years), participated in this study. All had normal or corrected-to-normal vision and were right-handed. Written informed consent was obtained from each participant after the nature of the study had been fully explained.

\section{Stimuli}

Stimuli were black drawings against a gray background, displayed on a Hitachi monitor, controlled by PsyScope on a personal computer (Macintosh G3) with a PsyScope button box (Cohen, MacWhinney, Flatt, \& Provost, 1993). The viewing distance was $80 \mathrm{~cm}$ and a central fixation cross that extended across a visual angle of $1.0^{\circ} \times$ $1.0^{\circ}$ was presented throughout the experiment. Bilateral stimuli consisted of two rectangles, which were displayed horizontally $3.56^{\circ}$ to the left and right and $1.28^{\circ}$ above the fixation point (to the inner edges). Each rectangle extended $1.07^{\circ}$ vertically and $0.71^{\circ}$ or $1.43^{\circ}$ horizontally for standards and $1.07^{\circ}$ for targets (i.e., square). Stimuli of different widths were selected for the left and right sides to make an asymmetric figure. The bilateral stimuli were either unconnected in the unconnected condition, or connected by a thin line $\left(0.05^{\circ}\right.$ wide $)$ in the thin/weakly connected condition or by a thick line $\left(0.50^{\circ}\right)$ in the thick/strongly connected condition.

These stimuli were randomly presented at $75 \%$ probabilities for standards and at equal probabilities for the other conditions. The durations of the bilateral stimuli were $100 \mathrm{msec}$, and the interstimulus intervals (offset to onset) were randomly varied between 300 and $500 \mathrm{msec}$ (6 steps, rectangular distribution).

\section{Procedure}

The participant was seated in a reclining chair in a soundand electric-shielded room and instructed to attend to either the left or right hemifield during the blocks and to press a button with the right thumb in response to the target presented in the attended field as accurately and quickly as possible. It was emphasized that they had to maintain fixation and to try not to move their eyes during the block. The attend-left and attend-right conditions consisted of 12 blocks, respectively, each consisting of 100 trials (except that there were 16 blocks of 120 trials for the first three participants in this experiment), which were alternated. The initial visual field to be attended was counterbalanced across the participants.
The experiment started with one to three practice blocks for each attention condition to stabilize task performance and eye movement.

\section{Recordings and Analyses}

The electroencephalogram (EEG) was measured using an electrocap (Electro-Cap International; Eaton, $\mathrm{OH}$ ) with 25 Ag-AgCl electrodes (Fp1, Fp2, F7, F3, Fz, F4, F8, T3, C3, Cz, C4, T4, T5, P3, Pz, P4, T6, O1, Oz, O2, PO7, PO3, POz, $\mathrm{PO} 4$, and $\mathrm{PO} 8$ according to the International 10-20 System), which were referenced to the nose. Blinks and horizontal eye movements were monitored with electrodes at the outer canthi of the eyes (horizontal electrooculogram [EOG]) and Fp2 and below the right eye (vertical EOG). The impedance of the electrodes was kept below $10 \mathrm{k} \Omega$. EEGs were filtered with a bandpass of $0.1-30 \mathrm{~Hz}$ and sampled at $200 \mathrm{~Hz}$.

Behavioral performance was measured, including the percentage of correct target detections (hits) and RTs for hits. Responses were scored as correct if they occurred within 300-1000 msec after a target in the attended location. Responses to other stimuli were classified as false alarms (FAs).

ERPs were averaged separately for each stimulus and for each attention condition. Further ERP analyses were conducted only for the standard stimuli to examine ERP components associated with visuospatial selection, whereas those associated with terminal decision and motor processes were separated (Hillyard \& Munte, 1984). Averaging epochs were $1000 \mathrm{msec}$, starting $200 \mathrm{msec}$ before the onset of the stimulus and ending $800 \mathrm{msec}$ poststimulus, while correcting for differences in the 200-msec prestimulus baseline. Automatic artifact rejection was applied to eliminate epochs contaminated above $75 \mu \mathrm{V}$. Epochs with incorrect responses and those immediately after responses (regardless of accuracy) were excluded.

\section{Results}

\section{Behavioral Data}

Figure 2 shows the behavioral results, with the attendleft and attend-right conditions collapsed. There were no significant differences regarding the attended directions. Hit and FA rates were generally low, which indicates that the participants carefully performed the present experimental task. There were main effects of connectedness for all behavioral indices $[\mathrm{Hit}, F(2,18)=9.1, p<.005$; RT, $F(2,18)=65.8, p<.001$; FA, $F(2,18)=5.7, p<.05]$. Planned comparisons showed that hit rates were higher and RTs were faster for the thin condition than for the thick condition $[t(9)=-3.5, p<.01$ (two-tailed); $t(9)=$ $-8.2, p<.001]$, but these did not significantly differ between the unconnected and thin conditions ( $p$ s $>.1$ ). In contrast, FA rates were higher for the unconnected 


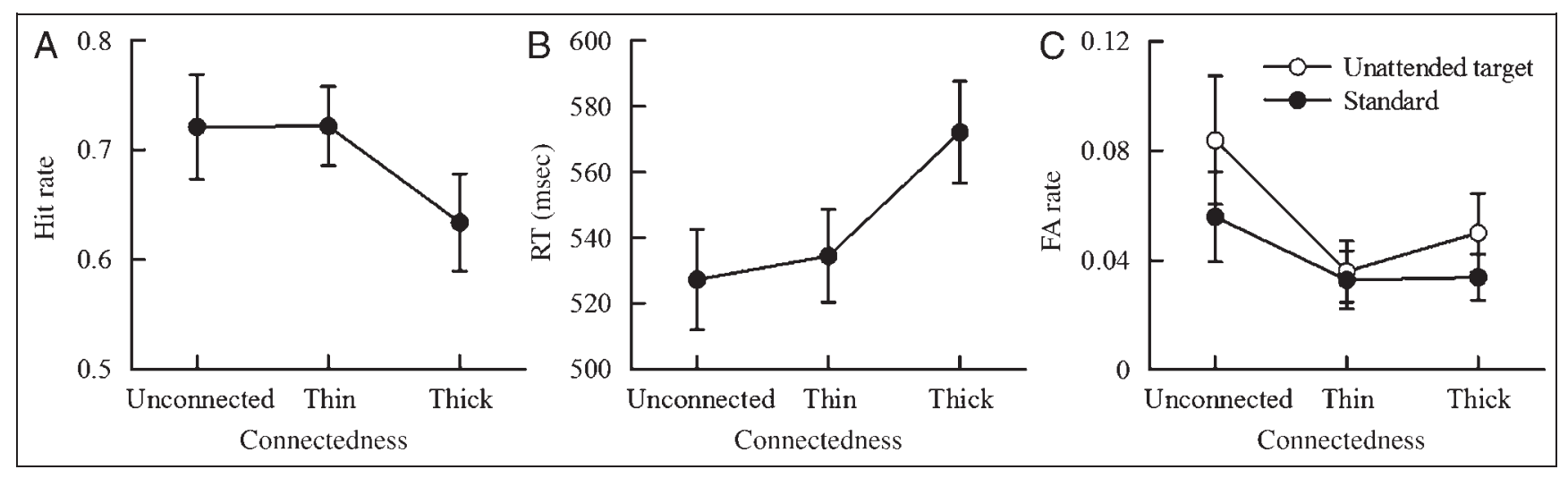

Figure 2. Behavioral results as a function of connectedness in Experiment 1. Data for the attend-left and attend-right conditions are collapsed. (A) Hit rates, (B) RT, and (C) FA rates. Error bars indicate standard errors of the mean.

condition than for the thin condition $[t(9)=-2.7, p<$ $.05]$ and did not differ between the thin and thick conditions $(p>3)$. There was also a main effect of type (unattended target, standard) for FAs $[F(2,18)=6.9, p<$ $.05]$, which showed that FAs increased for targets at taskirrelevant sides compared to the results for standards.

\section{ERP Data}

Figure 3A shows grand-averaged ERPs at the occipitaltemporal electrodes (PO7, PO8) that showed the greatest attention effects, the same as in our previous study (Kasai \& Kondo, 2007). Attention effects were clarified by difference waves between ERPs for the electrode sites ipsilateral and contralateral to task-relevant visual fields (Figure 3B), and quantified by mean amplitudes with latency windows of $80-140 \mathrm{msec}$ (poststimulus) for P1, 150-210 for N1, and 240-315 msec and 330-390 msec for N2pc. The measurements were subjected to repeated measures analysis of variance (ANOVA): The factors considered were attended visual field (left, right), connectedness (unconnected, weakly connected, strongly connected), and laterality (ipsilateral, contralateral) of the electrode sites relative to the attended visual field. The Greenhouse-Geisser correction was used to reduce the positive bias resulting from repeated factors with more than two levels.

P1 had a greater amplitude at contralateral than at ipsilateral sites for all connectedness conditions equally, as reflected by the main effects of laterality $[F(1,9)=$ $9.4, p<.05]$. In the N1 latency range, the difference ERPs were more positive as the extent of grouping increased, which was reflected by the interaction of laterality and connectedness $[F(2,18)=7.8, p<.01]$. To simplify further analyses, the attention effects (i.e., difference ERPs) were tested (Figure 3C), and the main effect of connectedness was significant $[F(2,18)=7.8$, $p<.01]$. Planned comparisons showed that the attention effect for the thin condition was significantly more positive than that for the unconnected condition $[t(9)=$ $-1.85, p<.05$ (one-tailed)], and that for the thick con- dition was more positive than that for the thin condition $[t(9)=-1.90, p<.05]$.

There were no statistically significant effects in the early N2pc latency range. The late N2pc latency range had a Laterality $\times$ Connectedness interaction $[F(2,18)=$ $7.9, p=.005]$, and the main effect of connectedness for the attention effects was significant $[F(2,18)=7.9, p=$ $.005]$. Planned comparisons showed that the attention effect for the thin condition was more positive than that for the unconnected condition $[t(9)=-3.77, p<.005]$ and that for the thin condition did not differ from that for the thick condition $(p>8)$.

\section{Discussion}

The lateralized attention effect of P1 indicates that the participants successfully directed their attention to the task-relevant visual field, whereas the positive deflection of the attention effects in the subsequent time ranges indicates that attention was spread or guided to the opposite side of the connected objects. This effect was observed for standard stimuli without behavioral responses, which suggests that the symmetrical structure may have confounded the results in the previous study where an attention-spreading effect was observed only for targets (Kasai \& Kondo, 2007).

Interestingly, two separate phases of the object-based attention effect were observed: The early phase (N1, 150$210 \mathrm{msec}$ ) was associated with the extent of perceptual grouping and the later phase (N2pc, 330-390 msec) was associated with object unity in terms of connectedness. This may suggest that spatial attention can be guided twice by different types of group/object representations.

On the other hand, behavioral indices were not necessarily consistent with the pattern of ERPs. However, they cannot be simply interpreted consistent with ERPs in response to standards because bilateral stimuli including target had a more symmetrical structure. Still, behavioral performance (Hit/RT) was worse for the thick condition than for the thin condition, consistent with the notion that the thick line made a stronger grouping 
and caused more attention-spreading over the other side of the group to decrease shape discrimination at the taskrelevant side.

Thus, the present results generally appear to show some evidence of attention-spreading or guidance based on object/group representations. However, one might argue that the present behavioral/ERP results were due to the interruption of sustained attention through the addition of connecting bars, that is, attentional capture or distraction by a change in luminance, rather than to attention-spreading over a group or an object. This possibility was tested in the next experiment.

\section{EXPERIMENT 2}

In this experiment, the extent of connectedness was varied by color unification of the connecting line (Figure 1), according to the notion that perceptual grouping is

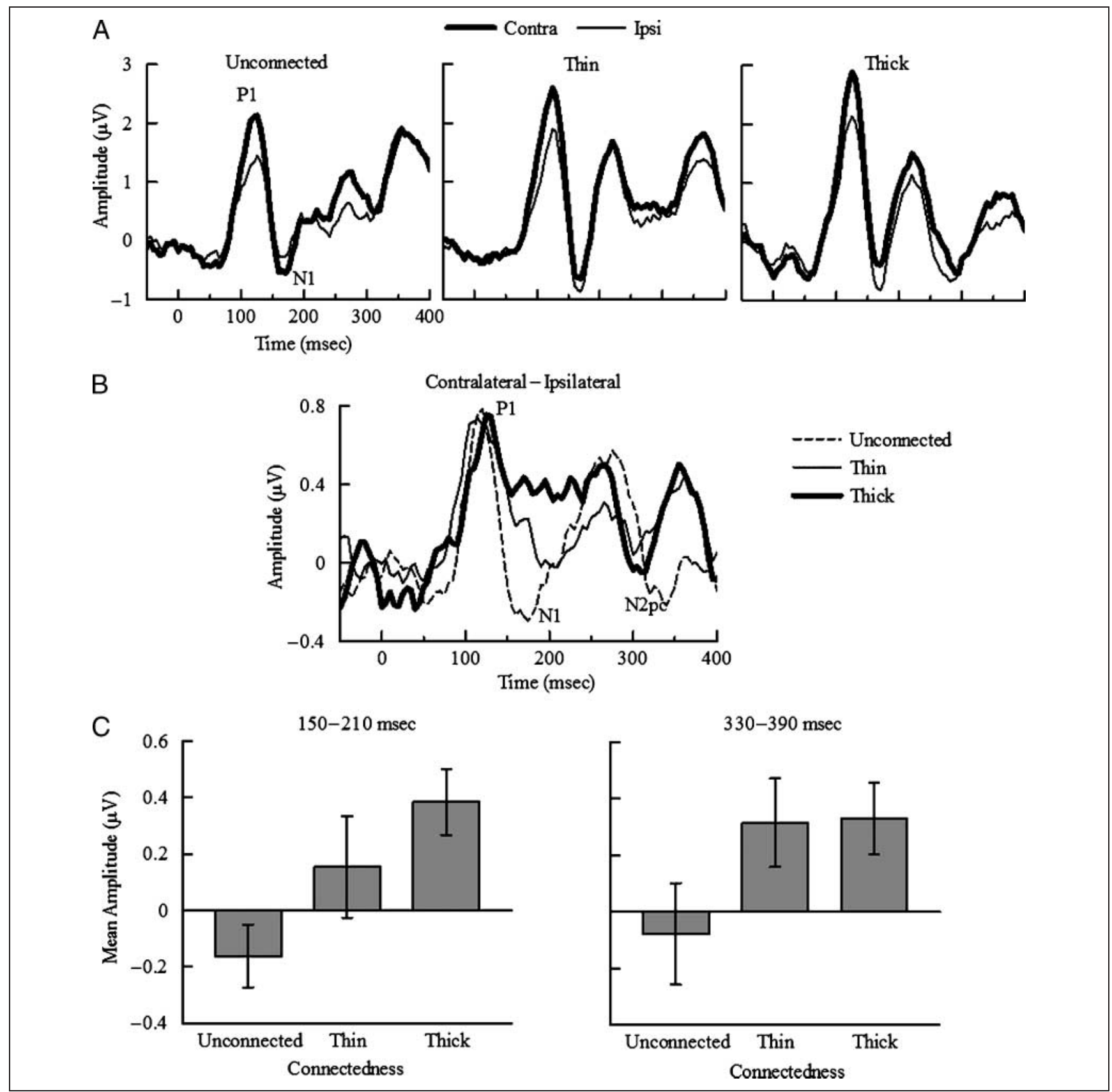

Figure 3. (A) Grand-average ERPs at the occipito-temporal electrodes (PO7, PO8) in Experiment 1. ERPs at hemisphere sites contralateral and ipsilateral to an attended visual field are overlapped, collapsing the left and right sites. Contra = contralateral; Ipsi = ipsilateral. (B) Difference waves for the attention effect, that is, ERPs at ipsilateral sites were subtracted from those at contralateral sites. (C) Mean amplitudes of the attention effect. Positive values indicate that attention was directed to the visual field opposite the task-relevant field. Error bars indicate standard errors of the mean. 
stronger for unified connectedness than for ununified connectedness (Palmer, 2003). Because the white and black regions were collapsed, the physical amount of stimuli was the same across the connected conditions. If the findings in Experiment 1 were due to a change in luminance due to the addition of connecting bars, a different pattern of results should be obtained in this experiment.

\section{Methods}

The methods were the same as those in Experiment 1, except as noted below.

\section{Participants}

Ten volunteers ( 5 women), aged 21 to 38 years (mean $=$ 27.6 years), participated in this study.

\section{Stimuli}

Stimuli were black or white drawings against a gray background. The bilateral rectangles were either unconnected in the unconnected condition, or connected by a line with the same width as that in the thick condition in Experiment 1 (i.e., $0.50^{\circ}$ ), which had either a different color than the bilateral rectangles in the ununified/weakly connected condition or the same color in the unified/strongly connected condition.

\section{Results}

\section{Behavioral Data}

Figure 4 shows the behavioral results. Connectedness had main effects for all behavioral indices [Hit, $F(2,18)=$ 18.9, $p<.001$; RT, $F(2,18)=25.5, p<.001$; FA, $F(2$, $18)=10.6, p<.005]$. Planned comparisons showed that although there was no significant difference in hit rates between the unconnected and ununified conditions $[p>$ .1 (two-tailed)], these were higher for the ununified condition than for the unified condition $[t(9)=3.5, p<.01]$. RTs seemed to increase as connectedness increased, as shown by a significant difference between the unconnected and ununified conditions $[t(9)=-5.1, p<.005]$, whereas this difference was marginal for the ununified and unified conditions $[p>.06]$. FA rates were higher for the unconnected condition than for the ununified condition $[t(9)=-2.9, p<.05]$, and there was no significant difference between the ununified and unified conditions $(p>.1)$.

\section{ERP Data}

Figure 5 shows the electrophysiological results in Experiment 2. Attention effects were quantified by mean amplitudes with latency windows of $80-140$ msec (poststimulus) for P1, 150-210 for N1, and 240-300 msec and 310-390 msec for N2pc.

P1 had a greater amplitude at contralateral than at ipsilateral sites for all connectedness conditions equally, as reflected by the main effects of laterality $[F(1,9)=$ $19.8, p<.005]$. In the N1 latency range, the difference ERPs were more positive as the extent of grouping increased, which was reflected by an interaction of laterality and connectedness $[F(2,18)=5.2, p<.05]$. The main effect of connectedness for the attention effects (i.e., difference ERPs) was significant $[F(2,18)=5.2$, $p<.05]$. Planned comparisons between the unconnected and ununified conditions failed to show statistically significant effects $(p>.1)$, whereas that for the unified condition was significantly more positive than that for the ununified condition $[t(9)=-2.97, p<.01$ (one-tailed)].

The early N2pc latency range had larger positive amplitudes, as reflected by a main effect of connectedness $[F(2,18)=4.08, p<.05]$, and this may involve the physical difference between the unconnected and connected conditions. There were no other statistically significant effects in this latency range. The late N2pc

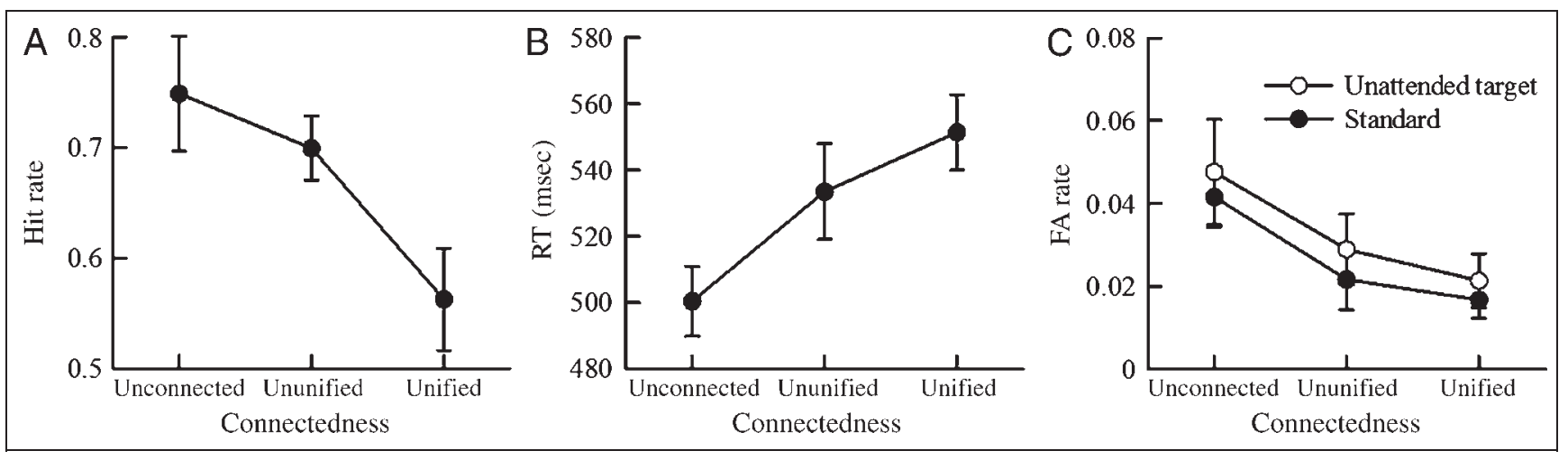

Figure 4. Behavioral results as a function of connectedness in Experiment 2. Data for the attend-left and attend-right conditions are collapsed. (A) Hit rates, (B) RT, and (C) FA rates. Error bars indicate standard errors of the mean. 


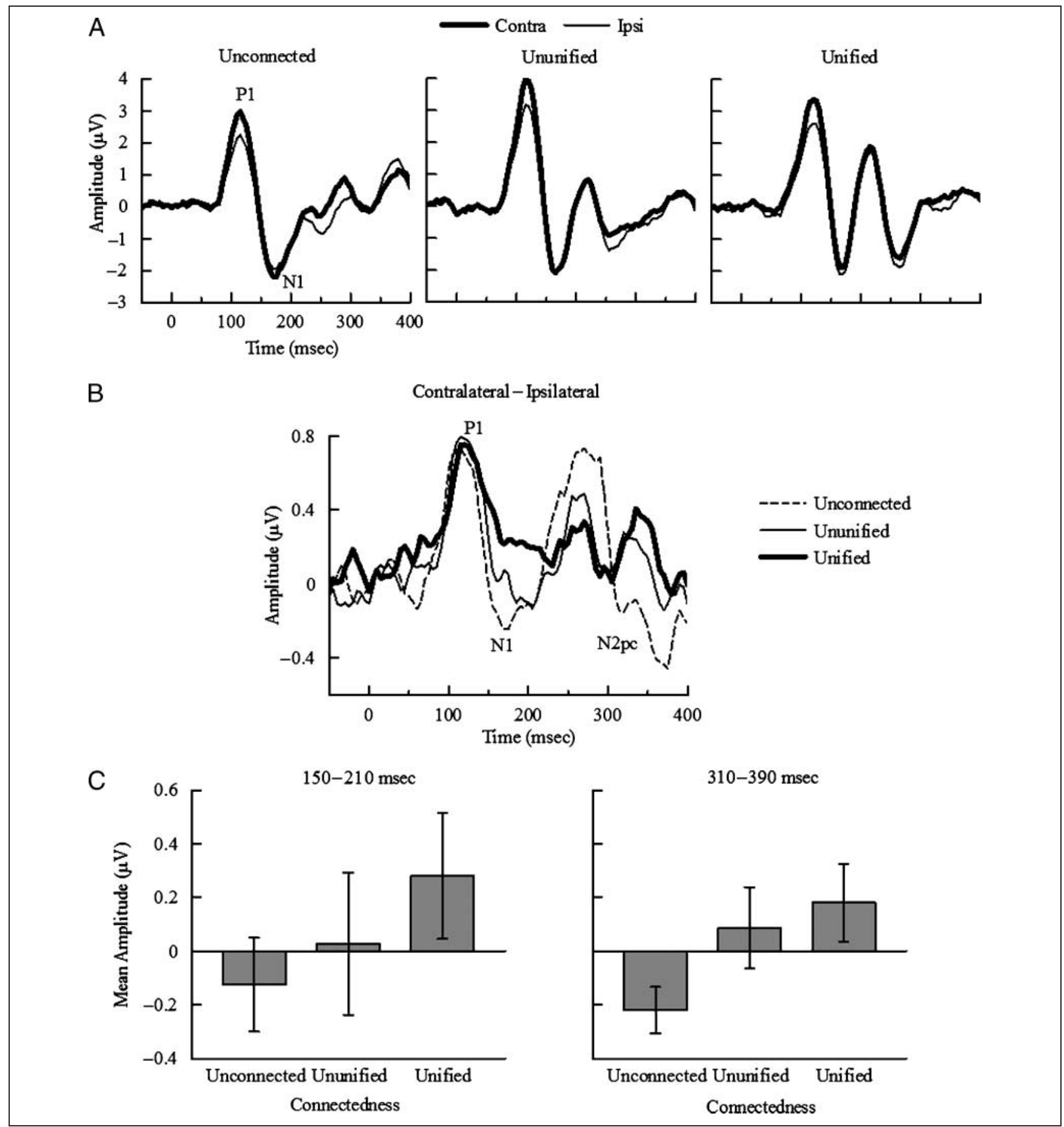

Figure 5. (A) Grand-average ERPs at the occipito-temporal electrodes (PO7, PO8) in Experiment 2. ERPs at hemisphere sites contralateral and ipsilateral to an attended visual field are overlapped, collapsing the left and right sites. Contra $=$ contralateral; Ipsi $=$ ipsilateral. (B) Difference waves for the attention effect, that is, ERPs at ipsilateral sites were subtracted from those at contralateral sites. (C) Mean amplitudes of the attention effect. Positive values indicate that attention was directed to the visual field opposite the task-relevant field. Error bars indicate standard errors of the mean.

latency range had a Laterality $\times$ Connectedness interaction $[F(2,18)=6.7, p<.01]$ and the main effect of connectedness for the attention effects was significant $[F(2,18)=6.7, p<.01]$. Planned comparisons showed that the attention effect was more positive for the ununified condition than for the unconnected condition $[t(9)=-2.97, p<.01]$, and that for the ununified con- dition did not differ from that for the unified condition $(p>.2)$.

\section{Discussion}

The patterns of the results in Experiment 2 were similar to those in Experiment 1. Behavioral performance (Hit/ 
RT) was worse and N1 attention effects decreased more for the strongly connected condition than for the weakly connected condition. N2pc attention effects and FAs were greater for the unconnected condition than for the connected conditions. These results do not support the view that the connectedness effects were due to attentional capture or distraction caused by the addition of connecting bars, but do suggest that the effects reflect obligatory attention-spreading guided by group/object representations.

\section{GENERAL DISCUSSION}

The goal of the present study was to investigate the timing of object-based spatial selection in response to object/group onset and the nature of representations. The results of the two experiments consistently revealed that two separate phases in ERP (N1, 150-210 msec poststimulus; N2pc, 330/310-390 msec) are associated with obligatory attention-spreading in response to connected objects. The present study is the first to show ERP manifestation of two processes or mechanisms of object-based spatial selection, and they differ with regard to representational levels of object processing: perceptual grouping in an early process, and the unity of connected objects in a later process.

\section{Two Processes of Object-based Spatial Selection}

This study found an object-based spatial attention effect in the N1 latency range, inconsistent to previous studies, in which the N1 attention effect was absent in the sustained-attention paradigm with rapid bilateralstimulus presentation (Woldorff et al., 2002; Heinze et al., 1990, 1994). This might be because grouping factors of bilateral stimuli (i.e., symmetry or common onset) nulled the N1 attention effect in the previous studies, or the early attention-spreading effect in this study was due to an early N2pc, rather than N1. Together with the notions that $\mathrm{N} 1$ reflects a gain control of incoming sensory signals in lateral occipital cortex (e.g., Martinez et al., 2006) and that N2pc reflects the suppression of competing stimuli in ventral extrastriate visual cortex (e.g., Hopf et al., 2002), further examinations with more electrodes and combinations of neuroimaging methods are required to reveal the neural bases of object-based spatial selection.

However, the present results suggest that an early spatial-selection mechanism is involved in object-based attention, as in previous studies with object-preview paradigms (Martinez, Ramanathan, et al., 2007; Martinez, Teder-Salejarui, et al., 2007; Martinez et al., 2006; He et al., 2004). The present study observed the objectbased attention effect of $\mathrm{N} 1$ as a decrease or polarity inversion of the lateralized spatial attention effect (i.e., amplitude difference between contralateral and ipsilateral hemifields relative to an attended location for bilateralstimulus array). On the other hand, the previous studies have shown that the N1 amplitudes increase for unattended locations that are connected to the attended region. Both results indicate that a location on the side opposite the attended location within an object/group is relatively enhanced at an early stage of visual processing. An original finding in this study is that the early attention effect was associated with perceptual grouping, rather than object unity.

The behavioral performance (Hits/RTs) also generally changed with the extent of grouping, consistent with studies that used the extent of the reduction in RTs as an index of the extent to which the parts are grouped or the extent of objecthood (Kawachi \& Gyoba, 2006; Marino \& Scholl, 2005; Palmer, 2003; Watson \& Kramer, 1999). In the present study, performance may have worsened as grouping increased because attentional resources were decreased for shape discrimination at the taskrelevant location due to attention guidance, similar to the findings with flanker-type paradigms (e.g., Kramer \& Jacobson, 1991; Driver \& Baylis, 1989). Thus, the present results suggest that the early cortical response in the N1 latency range reflects the interaction between spatial attention and object representations, which underlies behavioral responses, consistent with the findings in a previous ERP study (He et al., 2004). However, the present behavioral results were not object-based attention effects (i.e., difference between one-object and two-object conditions). The N1 attention effect may be a more direct index of grouping than the behavioral responses that are the final outputs of multiple stages of processing.

This study also found an object-based spatial attention effect in N2pc latency ranges, consistent with our previous study (Kasai \& Kondo, 2007), although the latency ranges were different (190-250 msec vs. 310/330 $390 \mathrm{msec}$ ). This may be because the timing of attentionspreading reflected by this potential can vary according to stimulus or task variables. Thus, the N2pc objectbased effect can occur regardless of the need for an explicit behavioral response for the stimuli (i.e., standard or target). This suggests a property common to the N1 object-based effect (Martinez et al., 2006): The N2pc effect also reflects an obligatory selection of entire object regions or an attention-spreading process (e.g., Driver \& Baylis, 1998), rather than a strategy for searching targets (Shomstein \& Yantis, 2002; but also see Goldsmith \& Yeari, 2003).

However, the object-based effect of N2pc is functionally different from that of N1. The amplitudes of N2pc changed with regard to whether objects were connected or unconnected, rather than based on perceptual grouping. Moreover, a similar pattern of results was observed for FAs. Although FA is not a typical behavioral objectbased attention effect, the present results may be considered according to the biased-competition model (Desimone \& Duncan, 1995). A segregated object that 
appeared within the attended location (i.e., unconnected condition) may become more salient by top-down biasing, compared with part of an object where attention is guided more to the opposite side of the object (connected condition). More salient stimuli would cause more FAs. This consideration meets the functional significance of N2pc (Hopf et al., 2002; Luck \& Hillyard, 1994) and suggests that two different mechanisms are involved in object-based spatial selection in response to object/group onset.

\section{The Nature of Object Representations for Attention}

There are some controversies regarding the nature of object representations on which attention operates, and ERP studies may help to clarify these issues. First, attention operates on space-invariant object representations as shown by an object-based attention effect for two overlapping objects (e.g., Duncan, 1984), whereas objects are fundamentally spatial representations, as spatial parameters affected object-based attention effects (e.g., Vecera, 1994). However, there may be at least two components with different sensitivities to spatial manipulations, depending on the task demands (Vecera \& Farah, 1994) or the timing of cues for targets (Awh, Dhaliwal, Christensen, \& Matsukura, 2001).

Because N1 is a typical component of spatial attention, we can infer that the N1 attention effect reflects the space-variant component in object-based selection. However, previous studies have also shown that N1 is associated with object-based attention in the case of overlapped surfaces at the same spatial location (Khoe, Mitchell, Reynolds, \& Hillyard, 2005; Pinilla, Cobo, Torres, \& Valdes-Sosa, 2001; Valdes-Sosa, Bobes, Rodriguez, \& Pinilla, 1998). Therefore, the early object-based effect of ERP occurs in both spatial and nonspatial cases, at least two-dimensionally, suggesting that it reflects processing at an intermediate stage from two-dimensional (2-D) retinal inputs to space-invariant or 3-D representations, consistent with the ERP attention effects in 3-D space (Kasai, Morotomi, Katayama, \& Kumada, 2003). On the other hand, N2pc might be associated with other spatial components in object-based attention. More systematic investigations on spatial factors may be useful for clarifying this point.

Second, the level of visual object processing, such as for line segments or perceptual/illusory objects, has been controversial (e.g., Avrahami, 1999; Moore, Yantis, \& Vaughan, 1998). The present results clearly showed two phases of object-based spatial selection, which suggests that multiple levels of processing may be involved and the critical conditions should be investigated to determine which levels become explicit in behavioral performance. On the other hand, an interesting point is that N1 is associated with object-based selection for both illusory objects (Martinez, Ramanathan, et al., 2007; Martinez, Teder-Salejarui, et al., 2007) and simple connected objects, as shown by the present results. This suggests that N1 may reflect the extent of perceptual grouping regardless of the type of grouping, or a process that occurs after all grouping factors are completed. However, because stimuli with different grouping factors can elicit ERPs in different time ranges (e.g., Han, Song, Ding, Yund, \& Woods, 2001), this should be further tested with other grouping factors, such as a common color or shape.

The present results may contribute to defining the term "object," which has been used intuitively in many studies (for a review, see Scholl, 2001). Although "group" and "object" are generally undistinguished (e.g., Driver \& Baylis, 1998), at least for object unification, this study argues that the concept of object should be distinguished from that of group in terms of hierarchical representations or different stages of processing. Interestingly, a neural model based on single-unit studies in monkeys assumes that multiple stages of visuocortical processing are involved in visual grouping via horizontal and feedback neural connections and suggests that these are also associated with biased competition (Roelfsema, 2006). Thus, converging evidence from electrophysiology studies in humans and monkeys may also be useful for revealing the multiple levels of object-based spatial selection in future studies.

Finally, the present ERP results shed light on the nature of the interaction between attention and spatial object representations. The results support the attentionspreading hypothesis at the point that obligatory sensory enhancement occurs over the whole region of an object/ group. However, this does not mean a simple spreading of attention. If attention homogenously spread over the entire region of an object or a group, the lateralized potentials should have been absent in highly grouped conditions. Rather, it seems that attention was guided to the side opposite the task-relevant side within the intermediate spatial representations of object/group. In addition, the extent of grouping did not change the speed of the attention shift but did affect the extent of biasing for the opposite side of the group. Such biased sampling of sensory information at an unattended location may contribute to activation or selection of the whole spatial region of the group or object, whereas its transient nature may be the basis of the limits and flexibility of the use of behaviors. Because attention may be closely linked to visual awareness, it would also be interesting to ask how the two attention-spreading processes are associated with our perception of the visual scene.

\section{Acknowledgments}

I thank two anonymous reviewers for their thoughtful suggestions on an earlier version of the manuscript. 
Reprint requests should be sent to Tetsuko Kasai, Faculty of Education, Hokkaido University, Sapporo, 060-0811, Japan, or via e-mail: tetsu@edu.hokudai.ac.jp.

\section{REFERENCES}

Avrahami, J. (1999). Objects of attention, objects of perception. Perception \& Psychophysics, 61, 1604-1612.

Awh, E., Dhaliwal, H., Christensen, S., \& Matsukura, M. (2001). Evidence for two components of object-based selection. Psychological Science, 12, 329-334.

Busse, L., Roberts, K. C., Crist, R. E., Weissman, D. H., \& Woldorff, M. G. (2005). The spread of attention across modalities and space in a multisensory object. Proceedings of the National Academy of Sciences, U.S.A., 102, 18751-18756.

Cohen, J., MacWhinney, B., Flatt, M., \& Provost, J. (1993). PsyScope: An interactive graphic system for designing and controlling experiments in the psychology laboratory using Macintosh computers. Behavior Research Methods, Instruments, and Computers, 25, 257-271.

Desimone, R., \& Duncan, H. (1995). Neural mechanisms of selective visual attention. Annual Review of Neuroscience, 18, 193-222.

Driver, J., \& Baylis, G. C. (1989). Movement and visual attention: The spotlight metaphor breaks down. Journal of Experimental Psychology: Human Perception and Performance, 15, 448-456.

Driver, J., \& Baylis, G. C. (1998). Attention and visual object segmentation. In R. Parasuraman (Ed.), The attentive brain (pp. 299-325). Cambridge, MA: MIT Press.

Duncan, J. (1984). Selective attention and the organization of visual information. Journal of Experimental Psychology: General, 113, 501-517.

Egly, R., Driver, J., \& Rafal, R. D. (1994). Shifting visual attention between-object and locations: Evidence from normal and parietal lesion subjects. Journal of Experimental Psychology: General, 123, 161-177.

Goldsmith, M., \& Yeari, M. (2003). Modulation of object-based attention by spatial focus under endogenous and exogenous orienting. Journal of Experimental Psychology: Human Perception and Performance, 29, 897-918.

Han, S., Song, Y., Ding, Y., Yund, E. W., \& Woods, D. L. (2001). Neural substrates for visual perceptual grouping in humans. Psychophysiology, 38, 926-935.

He, X., Fan, S., Zhou, K., \& Chen, L. (2004). Cue validity and object-based attention. Journal of Cognitive Neuroscience, 16, 1085-1097.

Heinze, H. J., Luck, S. J., Mangun, G. R., \& Hillyard, S. A. (1990) Visual event-related potentials index focused attention within bilateral stimulus arrays: I. Evidence for early selection. Electrophysiological and Clinical Neurophysiology, 75, 511-527.

Heinze, H. J., Mangun, G. R., Burchert, W., Hinrichs, H., Scholz, M., Munte, T. F., et al. (1994). Combined spatial and temporal imaging of brain activity during visual selective attention in humans. Nature, 372 , 543-546.

Hillyard, S. A., \& Munte, T. F. (1984). Selective attention to color and location: An analysis with event-related brain potentials. Perception \& Psychophysics, 36, 185-198.

Hopf, J. M., Boelmans, K., Schoenfeld, A. M., Heinze, H. J., \& Luck, S. J. (2002). How does attention attenuate target-distractor interference in vision? Evidence from magnetoencephalographic recordings. Cognitive Brain Research, 15, 17-29.
Hopf, J. M., Schoenfeld, M. A., \& Heinze, H. J. (2005). The temporal flexibility of attentional selection in the visual cortex. Current Opinion in Neurobiology, 15, 183-187.

Kasai, T., \& Kondo, M. (2007). Electrophysiological correlates of attention-spreading in visual grouping. NeuroReport, 18, 93-98.

Kasai, T., Morotomi, T., Katayama, J., \& Kumada, T. (2003). Attending to a location in three-dimensional space modulates early ERPs. Cognitive Brain Research, 17, 273-285.

Kawachi, Y., \& Gyoba, J. (2006). A new response-time measure of object persistence in the tunnel effect. Acta Psychologia, 123, 73-90.

Khoe, W., Mitchell, J. F., Reynolds, J. H., \& Hillyard, S. A. (2005). Exogenous attentional selection of transparent superimposed surfaces modulates early event-related potentials. Vision Research, 45, 3004-3014.

Kramer, A. F., \& Jacobson, A. (1991). Perceptual organization and focused attention: The role of objects and proximity in visual processing. Perception \& Psychophysics, 50, 267-284.

Luck, S. J., \& Hillyard, S. A. (1994). Spatial filtering during visual search: Evidence from human electrophysiology. Journal of Experimental Psychology: Human Perception and Performance, 20, 1000-1014.

Marino, A. C., \& Scholl, B. J. (2005). The role of closure in defining the "objects" of object-based attention. Perception E Psychophysics, 67, 1140-1149.

Marr, D. (1982). Vision. A computational investigation into the buman representation and processing of visual information. San Francisco, CA: W. H. Freeman.

Martinez, A., Ramanathan, D. S., Foxe, J. J., Javitt, D. C., \& Hillyard, S. A. (2007). The role of spatial attention in the selection of real and illusory objects. Journal of Neuroscience, 27, 7963-7973.

Martinez, A., Teder-Salejarui, W., \& Hillyard, S. A. (2007). Spatial attention facilitates selection of illusory objects: Evidence from event-related brain potentials. Brain Research, 1139, 143-152.

Martinez, A., Teder-Salejarve, W., Vazquez, M., Molholm, S., Foxe, J. J., Javitt, D. C., et al. (2006). Objects are highlighted by spatial attention. Journal of Cognitive Neuroscience, 18, 298-310.

Moore, C. M., Yantis, S., \& Vaughan, B. (1998). Object-based visual selection: Evidence from perceptual completion. Psychological Science, 9, 104-110.

Muller, N. G., \& Kleinschmdt, A. (2003). Dynamic interaction of object- and space-based attention in retinotopic visual areas. Journal of Neuroscience, 23, 9812-9816.

Palmer, S. E. (2003). Perceptual organization and groupings In R. Kimuchi, M. Behrman, \& C. R. Olson (Eds.), Perceptual organization in vision (pp. 3-43). Mahwah, NJ: Erlbaum.

Pinilla, T., Cobo, A., Torres, K., \& Valdes-Sosa, M. (2001) Attention shifts between surfaces: Effects on detection and early brain potentials. Vision Research, 41, 1619-1630.

Roelfsema, P. R. (2006). Cortical algorithms for perceptual grouping. Annual Review of Neuroscience, 29, 203-227.

Schoenfeld, M. A., Templemann, C., Martinez, A., Hopf, J. M., Sattler, C., Heinze, H. J., et al. (2003). Dynamics of feature binding during object-selective attention. Proceedings of the National Academy of Sciences, U.S.A., 100, 11806-11811.

Scholl, B. J. (2001). Objects and attention: The state of the art. Cognition, 80, 1-46.

Shomstein, S., \& Yantis, S. (2002). Object-based attention: Sensory modulation or priority setting? Perception $\mathcal{E}$ Psychophysics, 64, 41-51. 
Valdes-Sosa, M., Bobes, M. A., Rodriguez, V., \& Pinilla, T. (1998). Switching attention without shifting the spotlight: Object-based attentional modulation of brain potentials. Journal of Cognitive Neuroscience, 10, 137-151.

Vecera, S. P. (1994). Grouped locations and object-based attention: Comment on Egly, Driver, and Rafal (1994). Journal of Experimental Psychology: General, 124, 316-320.

Vecera, S. P., \& Farah, M. (1994). Does visual attention select objects or locations? Journal of Experimental Psychology: General, 123, 146-160.

Watson, S. E., \& Kramer, A. F. (1999). Object-based visual selective attention and perceptual organization. Perception E Psychophysics, 61, 31-49.

Weber, T. A., Kramer, A. F., \& Miller, G. A. (1997). Selective processing of superimposed objects: An electrophysiological analysis of object-based attentional selection. Biological Psychology, 45, 159-182.

Woldorff, M. G., Liotti, M., Seabolt, M., Busse, L., Lancaster, J. L., \& Fox, P. T. (2002). The temporal dynamics of the effects in occipital cortex of visual-spatial selective attention. Cognitive Brain Research, 15, 1-15.

Woodman, G. F., \& Luck, S. J. (1999). Electrophysiological measurement of rapid shifts of attention during visual search. Nature, 400, 867-869. 